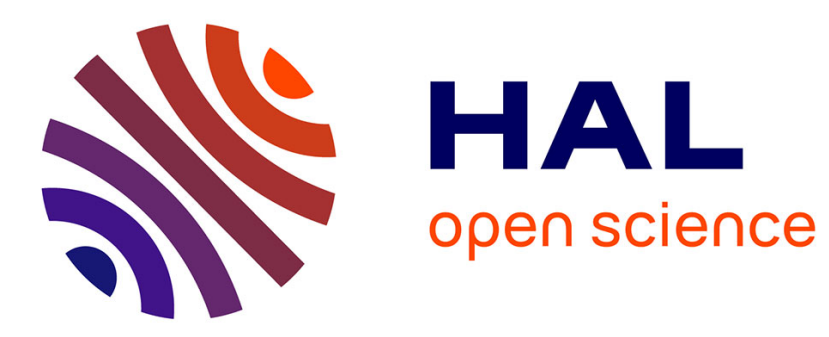

\title{
Gustav Bergmann et les complexions meinongiennes
}

Bruno Langlet, Jean-Maurice Monnoyer

\section{To cite this version:}

Bruno Langlet, Jean-Maurice Monnoyer. Gustav Bergmann et les complexions meinongiennes. Gustav Bergmann: Phenomenological Realism and Dialectical Ontology, 4, pp.201 - 228, 2009, 9783868380354. hal-02397039

\section{HAL Id: hal-02397039 \\ https://hal-amu.archives-ouvertes.fr/hal-02397039}

Submitted on 6 Dec 2019

HAL is a multi-disciplinary open access archive for the deposit and dissemination of scientific research documents, whether they are published or not. The documents may come from teaching and research institutions in France or abroad, or from public or private research centers.
L'archive ouverte pluridisciplinaire $\mathbf{H A L}$, est destinée au dépôt et à la diffusion de documents scientifiques de niveau recherche, publiés ou non, émanant des établissements d'enseignement et de recherche français ou étrangers, des laboratoires publics ou privés. 


\title{
Gustav Bergmann et les complexions meinongiennes
}

\author{
BRUnO LANGLET \& JEAN-MAURICE MONNOYER
}

(Preprint - Published in Langlet \& Monnoyer (eds), Gustav bergmann: phenomenological realism and dialectical ontology, Ontos verlag 2009.)

\begin{abstract}
«You believe, not only that the phenomenological and the dialectical context must be clearly separated, but also that an accurate grasp of their interplay is indispensable for doing ontology. I agree. », Realism, p. 69
\end{abstract}

G. Bergmann a entretenu avec Meinong des rapports qui ne sont pas faciles à débrouiller. En 1967, Meinong est présenté comme un interlocuteur " historique »: ce qui explique le sous-titre de l'œuvre dédiée à la " glorious memory» du philosophe de Graz, Realism : a Critique of Brentano and Meinong (notamment dans les chapitres 18 et 20, le chapitre 18 reprenant la thèse du chapitre 13 et la condamnation du « réisme » chez les deux auteurs) ${ }^{1}$. Bergmann y garde ses distances vis-à-vis de Findlay ${ }^{2}$, le gardien de la doctrine, p. 417 ; il se livre à un examen presque exhaustif de ses premiers essais, et prioritairement de Sur les objets d'ordre supérieur, mais sans tenir compte aucunement de l'évolution de Meinong; il discute même des changements entre les deux versions de Über Annahmen (1902-1910), à défaut de les expliquer vraiment.

Comme l'a très bien montré Venanzio Raspa, Bergmann a lu Meinong «sans méthode interprétative », et il lui fait « violence » ${ }^{3}$. Bien que reconnaissant son « flair incomparable», Bergmann critique son style «diffus"; il ironise sur la poursuite de ces «papillons phénoménologiques » qu'il chasserait pour son seul plaisir (III, ibid.. p. 342); de même, se plaint-il de sa terminologie "excentrique », sauf qu'il la réutilise et la détourne. Bergmann appelle la Relation de Meinong " connection », et se réserve justement le terme de " relation » (p. 344). Pourtant, la fin du livre adopte un verdict beaucoup plus nuancé. Bergmann révèle que sur le fond Meinong a compris la "structure relationnelle » présentée dans l'expérience. The most memorable Don Quixote of a great cause entraine soudain son affection et son admiration (pp. 440-41). Le tout dernier $\S$ de Realism annonce effectivement une autre lecture, et paraît dessiner une forme anticipée de retour en grâce. Cela ne signifie en rien, selon nous, que Bergmann soit devenu ensuite à proprement parler « meinongien ». Mais il est vrai que certaines des thèses de New Foundations of Ontology (rédigé en 1977, et édité en $1992^{4}$ ), ou développées dans son époque tardive, pourraient être lues comme proches de celles que Meinong a préfigurées ou comme des conséquences objectives de la pensée de Meinong.

${ }^{1}$ : Collected Works, Vol. III, Ontos Verlag, 2005.

2 : J. N. Findlay, Meinong's theory of objects and values, Oxford Clarendon Press, 1963.

${ }^{3}$ : Venanzio Raspa, «' '...The most memorable Don Quixote of a great cause', Bergmann's critique of Meinong », in Fostering the Ontological Turn, Rosaria Egidi, G. Bonino (eds), Ontos Verlag, Heusenstamm, 2008, pp. 201-228.

${ }^{4}$ : New Foundations of Ontology, Edited by William Heald, Foreword by Edwin E. Allaire, The University of Wisconsin Press, 1992 (=NFO). 
Nous suivrons donc l'hypothèse générale qu'a soutenue Rosaria Egidi ("Bergmann intends to carry out the Meinongian programm $»^{5}$ ) : pour elle, la critique du « représentationalisme », qui suit celle du réisme et du nominalisme (un résultat où Bergmann dit lui-même que "Meinong [realist] came agonizingly close », p. 340), ne pouvait que le conduire à revenir vers Meinong, un retour qui celui-là n'est plus historique. La position de Bergmann est intéressante et topique, indépendamment de sa sophistication. Elle se distingue de celles des autres interprètes qui ont entrepris par la suite de relire Meinong (R. Routley, R. Chisholm, T. Parsons, E. Zalta, R. Grossmann, K. Lambert, F. Nef, D. Jacquette).

\section{1/ Le « Meinong 》 de Bergmann}

L'essentiel dans cette réhabilitation inattendue ne peut pas se dire en quelques mots. Ou plutôt, l'article lumineux qu'a donné E. Tegtmeier : «Meinong's Complexes » pourrait servir de guide contre d'éventuels égarements dans cette « reconstruction rationnelle » de Meinong qu'a tenté de faire Bergmann ${ }^{6}$. On peut néanmoins entrer en matière par un autre biais, à cause simplement d'une formulation audacieuse et d'un revirement qu'effectue Bergmann dans les "early seventies », comme il le déclare très ouvertement devant H. Hochberg. Le point n'est pas seulement exégétique et terminologique. L'affaiblissement tardif du rôle crucial qu'aura joué, dans l'ontologie de Bergmann, le nexus d'exemplification - lequel se transforme du tout au tout dans les New Foundations, puisqu'il n'est plus un «constituant» comme il l'était encore dans Realism ${ }^{7}$ —, est solidaire d'un changement de «prototype » et des nouveaux canons de l'ontologie bergmannienne. Ce changement l'entraîne à l'adoption des fonctions dites «meinongiennes » (les M-Functions) (NFO, p.131): un «tribut» que Bergmann veut rendre « à cet esprit tortueux, aussi profond que subtil » (id.). Il désigne ainsi, en opposition aux fonctions "frégéennes" insaturées, ces fonctions qui conservent une relation basique avec des existants, par deux actes simultanés de prise de conscience directe de leur diversité. Ainsi renommées et correspondant à un meaning-nexus, les fonctions meinongiennes engageraient donc à l'existence des complexes, leurs fondements - entre les « choses » (les faits), et les " classes » —, mais en un sens il est vrai exactement contraire à celui que Bergmann a longuement défini dans Realism, quand il faisait la critique de la « complétion» de la complexion. Que s'est-il passé ? Bergmann n'avait-il pas opposé l'ontologie des fonctions et l'ontologie des complexes? H. Hochberg avoue qu'il lui semble que cette nouvelle ontologie est «baroque ». Une propriété du «contenu » formerait une circonstance donnée, couplée avec son intention ou son Objectiv. Les déterminés (couleurs, sons) s'accrocheraient ensemble (clinging to), hors du making de l'exemplification pour constituer un complexe. On pourrait certes, comme l'a indiqué Venanzio Raspa, suspecter d'emblée l'usage très libre des mots : «Komplex» et "Relation », qui avaient été transposés de l'allemand en anglais, et qui ne retrouvent pas ici leur vrai place. Mieux vaut s'en tenir à l'illustration directe qui est proposée de la M-Function. Par exemple, une quinte entre deux notes existe sans conjonction, sans terme supplémentaire. L'exemple peut sembler trop

\footnotetext{
5 : Rosaria Egidi, « Il Meinong di Gustav Bergmann », in C. Barbero, V. Raspa eds, in Il Pregiudizio a favore del Reale, Rivista di Estetica, XLV, Ns, n³0, Torino, 2005, pp. 54-70. Ce faisant nous ne donnons pas tort à l'examen minutieux des distorsions qu'a relevées V. Raspa en défenseur vigoureux de Meinong.

${ }^{6}$ : E. Tegtmeier, « Meinong's complexes », The Monist, $83: 1,2000$, pp. 89-100.

7 : H. Hochberg, The Positivist and the Ontologist, Bergmann, Carnap and Logical realism, Rodopi, Amsterdam, 1999, p. 160.
} 
pauvre pour explorer les Two-in-ones (inseparable compound of two simples), mais Bergmann — comme d'ailleurs Meinong - y reviennent souvent ${ }^{8}$.

Si ces M-functions jouent un rôle d'abord assez allusif dans l'économie générale d'une ontologie réaliste, elles sont donc en affinité étroite avec ces circumstances mystérieuses, qui sont effectivement présentées ailleurs, et principalement dans Notes on Ontology (1981), comme devant se substituer à la notion du non-relational Tie. Les circonstances « se fondent» dans le complexe tout en demeurant des éléments du complexe sous un autre rapport : celui de leur "diversité » (p. 139). Si nous comprenons bien, chaque note est une circonstance désignée (telle note), l'intervalle de quinte est lui aussi une circonstance, l'intention mentale qui vise la propriété de relation complexe : « être une quinte », en est une autre9.

L'indication diacritique $\mathrm{du}$ «M $\mathcal{M} »$ dans les Notes on Ontology qui signale le dernier mot de Bergmann sur le sujet, semble également être une extrapolation des M-functions. C'est une indication infiniment plus souple; elle ne tient lieu de «rien d'existant dans le monde »: ses arguments sont ceux d'une «fonction de signification» (p. 331), comme il en va dans le Tractatus pour le sich verhalten des faits «subsistants » qui ne sont pas présentables dans le langage idéal. Cette fonction ne réfère pas, mais elle peut aussi valoir cependant pour les dyades de toutes sortes réunissant un universel et un particulier : elle apparaît donc assez éloignée, à première vue, de cette allusion qui est faite cursivement dans New Foundations aux « objets d'ordre supérieur » comme à leur modèle structural (NFO, 131). Des fondements (les notes) au fondé (la quinte), Bergmann dit alors, suivant ici directement Meinong, "si les premiers sont là, la seconde est là eo ipso ». Quand l'on y regarde de près, il est clair cependant que les présentations signifiantes sont d'abord intentionnelles (puisqu'il y a une dyade séparable et une fonction inséparable d'elle, de même que chez Meinong il y a une différence entre le complexe donné et la pensée du complexe factuel qui le fait subsister), en sorte qu'une problématique déterminée du fondement (foundation) conditionne le mode de la liaison en dehors d'une relation linéaire ou temporelle ${ }^{10}$. Plus subtilement encore, Bergmann ajoute que le meaning-nexus est lui même une nouvelle sorte de circonstance.

Mais il faut d'abord revenir à la critique de Meinong par Bergmann dans Realism avant d'anticiper sur l'occurrence d'idées meinongiennes dans New Foundations. La position du concept de nexus, dans l'œuvre majeure de 1967, jouait un rôle décisif en regard des points de l'ontologie de Meinong sur lesquels Bergmann faisait porter sa critique. Le nexus devrait occuper en principe dans Realism le rôle d'un "fondement» ontologique des complexes (grounding): mais c'est un fondement subsistant (pp. 7-9), qu'il considèrera justement

\footnotetext{
${ }^{8}$ : Il faut déjà noter la différence avec Realism où la tierce et la quinte sont des universaux (Ontos Verlag, T. III, p. 344). Mais on peut ensuite lire chez Bergmann une longue analyse de ces questions qui rejoint la problématique des « tropes », puisque l'exacte similarité entre deux paires de quintes différentes se distingue de la similarité entre deux quintes obtenues à partir de deux notes identiques.

${ }^{9}$ : Ce mode d'accrochage oblique («slantwise ») des 3 plans dépend de ces deux sortes fondamentales d'entités que Bergmann isole dans sa dernière période: les determinates (indépendants et séparables), et les subdeterminates, qui ne sont pas séparables et ne sont pas explicitement des objets de l'acte intentionnel. Les subdeterminates ne peuvent être visés que slantwise, dans leur union avec d'autres entités formant un complexe qui est signifié par un acte intentionnel. Les déterminés sont des choses qui ne sont pas absolument simples, des complexes ou des classes. Les entités absolument simples sont sous-déterminées ou sont des subsistants, tels les connecteurs et les quantificateurs dans le langage idéal (Bergmann, 1981, Ontos Verlag, Collected Works, Vol. II, p. 322). Dans l'exemple donné, les circonstances sont imbriquées dans le complexe sous-déterminé par ces dernières.

10 : Frederic Nef (dans ce même volume) jette une lumière nouvelle sur la différence entre «lien» et « relation».
} 
ensuite comme un "sous-déterminable ». De plus, en tant que « constituant», comme on l'a vu ci-dessus, le nexus n'est pas un composant. Il remplace la forme de collection propre aux fonctions qui ne peuvent pas revendiquer un tel statut et ne seraient rien d'autre que des règles de " coordination ${ }^{11}$. Le nexus nous dispense d'une autre liaison pour être coordonné aux autres constituants du fait, alors que ces derniers ont toujours besoin de lui. Dans cette définition minimale, il n'y a rien en droit qui " supporte » les complexes réductibles à des circonstances ou assimilables à des faits. D'autant que les choses (ordinaires) sont des complexes improprement dits. En quoi le nexus ou le tie pourrait-il alors servir de fondement pour des complexes « authentiques »? La question mérite d'être posée. Ce qui est sûr est que dans les New Foundations, les fonctions (meinongiennes) servent à rétablir la notion de « fait $»^{12}$, alors que Bergmann avait admis que le monde de Meinong ne contenait pas de faits.

Si les positions assumées par le Bergmann de la late ontology ne correspondent plus vraiment à celles qu'il avait critiquées chez Meinong dans Realism, notre hypothèse permettrait de penser que Bergmann a ré-incorporé à sa philosophie des traits meinongiens qui avaient été plus ou moins passés sous silence, voire même évincés de la reconstruction fournie pas Realism. Nous ne prétendons pas offrir ici la seule clé de la lecture de l'évolution de Bergmann, car elle ne gouverne ni l'évolution du philosophe de l'Iowa, ni la permanence de certaines de ses positions. Son intransigeance sur la question des universaux et la critique persistante adressée à « Meinong-le-nominaliste » en sont la preuve. Comme on le verra, cette même question ne peut être rendue intelligible que sous l'éclairage de la pensée de Russell ; elle s'enracine aussi dans le rapport lui-même tortueux que Bergmann entretient avec Carnap.

La réprobation de Bergmann dans Realism concerne bien, à coup sûr, le sujet le plus central : la question de la nature de la relation qui fait qu'un collectif " objectif » [Objektiv Kollektiv] se distingue d'un complexe réel. Meinong aurait une ontologie « inadéquate » à cet égard, d'abord à cause de son refus des universaux. Bergmann s'étonne ensuite de voir dans les solutions de Meinong une ontologie implicite de la fonction, dont il se surprend que le philosophe de Graz ne l'exploite pas, malgré sa finesse ontologique. Le caractère implicite de cette ontologie de la fonction la distingue déjà de l'ontologie de la fonction (explicite) de Frege. Comme le dit Tegtmeier, il est très douteux qu'elle soit fidèle à Meinong (id., p. 94). D'abord, si les complexes de Meinong sont des objets d'ordre supérieur, ils sont dépendants et incomplets (unselbstängig et unfertig) : ils restent à mi-chemin de leur actualisation et de leur remplissement potentiel. (Ainsi une hauteur de ton pour une note peut se combiner avec une autre hauteur de ton pour produire une autre quinte ou produire une tierce). Ensuite, leur défaut d'unité est patent : la manière dont Meinong envisage le «fait » que le superius soit là eo ipso dès que les inferiora sont présents, est obtenu dans Realism selon une conception de l'intégration opérée par des relations internes qui ne peuvent en réalité rien connecter du tout. On sait qu'elles n'ont de support ontologique en tant que relations que dans la « nature » de leurs termes. Que la tonique et la dominante se rencontrent dans la quinte, ne crée en rien, pour Bergmann, l'entité-quinte : ce sont là des relations externes à la hauteur pure des notes, et c'est pourquoi elles définissent, par exemple — anecdotiquement, mais au sens littéral du mot - le style musical hérité de Haydn. Qu'une quinte soit plus haute qu'une autre quinte, forme aussi, entre deux quintes, une collection, et même une classe. Mais les fondements qui nous sont présentés ne sont jamais que les quintes (les complexes donnés avec les notes), en plus des rapports subsistants entre les intervalles.

\footnotetext{
${ }^{11}$ : G. Bergmann, Realism, The University of Wisconsin Press, 1967, p. 7. Selected Works II, Ontos Verlag, p. 7.

${ }^{12}$ Ibid, pp. 128-129.
} 
Dans New Foundations le renversement est total (presque «dramatique », souligne Hochberg) : les fonctions dites meinongiennes disqualifient les fonctions frégéennes et sont émancipées du modèle des relations internes. Plus important sans doute, ce que Bergmann y appelle le «principe de présentation », est doté d'une importance majeure en prenant de fortes résonances meinongiennes : ce principe énonce que tout ce qui fait l'objet d'une présentation « existe» (en incluant le Sein et le Sosein). Dédoublant le principe de présentation avec le principe d'acquaintance, hérité de la lecture de Russell - et d'après lequel toute acquaintance avec un universel est intimement solidaire d'une acquaintance avec un particulier, un item ou un « individuateur » - Bergmann pense aussitôt que les universaux doivent, eux aussi, faire l'objet d'une «présentation». Il adopte ce faisant et transforme un principe meinongien, même si l'accusation de nominalisme envers Meinong persiste.

\section{2/ Le problème de la relation « ajoutée »}

Pour comprendre ce changement de lecture, il nous faut d'abord souligner quel a été son point de départ. Dans Realism, Bergmann affirme que Meinong aurait prétendu fonder les objets de son ontologie en ajoutant un particulier spécial à une " collection de particuliers ». L'ajout en question est celui de la relation, qu'en l'occurrence Bergmann décide d'identifier comme un particulier de plus. Il est vrai que cette relation est désignée par Meinong comme ce qui « s'ajoute ${ }^{13}$ » (hinzukommt), mais de manière très caractéristique, c'est-à-dire lorsqu'un complexe est déjà unifié ou actuel ou lorsque que la relation « fait partie d'un tout » qui ne se résorbe pas en elle: bref, quand nous avons un complexe duquel les constituants sont réellement connectés et non pas seulement juxtaposés ou réunis ${ }^{14}$.

Au regard de la distinction existant entre un collectif objectif (cluster) — une chose ou une collection - et un complexe réel (complexion), Meinong est déjà plus proche de la problématique de Bergmann que ce dernier n'entend y consentir. S'ils traitent d'un problème apparenté, il reste que la « solution » de Meinong reste pour Bergmann inadaptée. Bergmann conteste la pertinence de «l'ajout», absolument impropre selon lui à fonder ontologiquement la distinction entre les deux types de complexités. Il soutient classiquement que si une relation est ce qui fait qu'un complexe «est » connecté, il faut expliquer comment cette relation se relie à ses relata. Or, elles ne peuvent faire cela par elles-mêmes : il faudrait toujours une relation supplémentaire, un connecteur de plus, pour justifier de la « relationnalité » de la

\footnotetext{
13 : « Über Gegenstände höherer Ordnung und deren Verhältnis zur inneren Wahrnehmung », Zeitschfrit für Psychologie und Physiologie der Sinneorgane, 21 (1899), pp. 187-272 : Gesammtaushabe, Vol II, ed. by R. Haller, Graz, Akademische Druck-u.Verlaganstalt, p. 391. Trad. Fr. G. Frechette, «Sur les objets d'ordre supérieur et leur rapport à la perception interne », in G. Frechette \& D. Fisette (dirs), A l'école de Brentano, Vrin, 2007, p. 272.

14: On retrouve dans Über Annahmen, cette même différence dans l'identification des complexes qui se démarque de la juxtaposition des composants. Les choses sont aussi beaucoup plus compliquées dans l'évolution de Meinong, car Meinong regarde d'abord à la fois le complexe [aRb] et la relation individuelle (a) R (b) comme des objecta. Il y a une relation d'équivalence logique entre l'objectif : que [aRb] existe et l'objectif : que (a) R (b) subsiste. Mais encore faut-il, selon Meinong, « que $a$ soit dans la relation $\mathrm{R}$ avec $b$ » - ce qu'il appelle justement Komplexion : celle-ci étant à la fois une réunion par la pensée et une conscience de cette réunion. Meinong oppose déjà Relation et Komplexion au $\S 5$ de Sur les objets d'ordre supérieur.
} 
relation (tel que s'exprime alors Bergmann). La solution de Meinong, exposée de la sorte, ne permettrait pas d'écarter le problème de Bradley : il est impossible d'y voir le fondement ontologique de la connexion adéquate entre constituants d'un complexe — soit un fondement qui serait ce qui « fait » que le complexe existe comme tel.

Selon Bergmann toutefois, Meinong échapperait - en principe - à cette critique. La relation "ajoutée ", pourrait recevoir le statut d'une fonction distincte si elle pouvait être considérée de manière séparée du tout qu'elle unifie en tant que fonction (simple), et donc en prenant les éléments du complexe comme arguments. Or, si le statut de la fonction permet ainsi d'échapper au danger bradleyien de la régression, c'est néanmoins précisément cette seule fonction comme "entité» (supposée toujours implicite chez Meinong) qui l'empêcherait d'avoir une complex-ontology, puisque la fonction devrait être "à elle-même » son propre fondement. D'une confusion (blurred grasp), on passe à un gap (une absence). Cette absence de fondement ontologique explique que Meinong échoue à connecter réellement les entités dans un «fait»: le nexus lui fait défaut, et seules les propriétés de ce dernier sont décisives pour obtenir un fait. Bref, si la seule « relationnalité » disponible pour Meinong est interne, c'est un échec selon Bergmann. Par conséquent, la relation supraadditive ou complexive entendue au sens de ce dernier - si l'on peut user de ce terme contradictoire pour tenter de la comprendre - ne saurait avoir de fondement ontologique adéquat (tandis qu'elle peut en avoir un chez Frege). Lorsqu'elle est capable en effet de colliger ou de lier et une fois qu'elle est entendue comme une fonction, elle perd son grounding et se soustrait au modèle pertinent de la complex ontology.

En réalité, cette critique échoue selon nous pour une seconde raison. Elle échoue parce que Meinong ne se met pas en quête d'un fondement ontologique lorsqu'il met en avant l'ajout d'une relation caractéristique pour les complexions. Ces dernières sont présentées sous l'aspect d'objets d'ordre supérieur. Sur le plan strict du fondement, rien n'autoriserait à retraduire dans un autre idiome, un idiome fonctionnel, l'ontologie de Meinong.

Dans Realism, Bergmann procède ainsi à une analyse très fine de l'ontologie que Meinong « devrait» assumer. Son verdict est que les particuliers peuvent bien s'agglutiner, mais sans jamais former un complexe intégré :

« Let $\alpha$ be an object; $\left\{a_{1}, \ldots, a_{\mathrm{n}}\right\}$, the collection of its particulars. Meinong accepts the ultimate premiss that the collection as such does not exist. Thus he knows that unless he does something about it, he will be left without objects. This is his strength. His weakness lies in what he does. Roughly speaking, he adds a $(n+1)$ st particular, of a very special status. But he fails to see that, even though the particular he adds exists if and only if $\alpha$ exists, $\alpha$ itself literally still doesn't exist, even if the particular does ${ }^{15}$ ».

Il est difficile de distinguer dans cette présentation si le particulier « au statut spécial» de Meinong est la relation elle-même ou l'objet d'ordre supérieur. Le résultat pour lui « ne fait pas de différence ». Il n'y aurait pas ici d'objet $\alpha$ (existant factuellement) qu'on puisse nommer complexe. La thèse de Bergmann est que Meinong s'en tient à une tentative de coordination entre des éléments et qu'il ne dispose pas ontologiquement de la possibilité d'avoir des objets. La relation serait quelque chose de superflu pour la collection des particuliers : elle serait « ontologisée » comme une internal connection (p. 338) Or, on ne saurait en toute rigueur lire cet ajout comme un geste gratuit de Meinong et comme s'il

\footnotetext{
15 : Realism, p. 337.
} 
s'agissait pour lui de "construire » des objets. Ce n'est pas le fondement ontologique que recherche ici Meinong. Le caractère impressionnant et fouillé de la critique de Bergmann laisse apparemment peu de place pour une contestation de son bien-fondé. Il ne nous reste que la possibilité d'y voir une modification du sens de « l'ajout » dont Meinong fait mention.

La reconstruction de Bergmann est telle cependant qu'elle écrase complètement les traits saillants du Meinong historique : la relation dont parle Meinong n'est pas une nouvelle entité introduite en vue d'unifier de nouveaux éléments " dans » une entité hétérogène. Elle ne vise pas à créer la connexion : elle est plutôt ce qui est appréhendé comme étant là lorsqu'il y a une complexion. Elle lie des constituants lorsqu'ils sont unifiés dans leur présentation et n'est donc pas ce qui permet de les unifier. L'ajout est analytique. Meinong va de la théorie de l'objet vers la postulation des objets d'ordre supérieur. Un complexe unifié ou intraconnecté est un objet d'ordre supérieur; son contraire étant une collection objective. Les modèles inspirateurs et critiqués par lui d'objets d'ordre supérieur ont bien été (et demeurent en arrière-plan) les Gestaltquälitaten, depuis l'article Zur Psychologie der Komplexionen und Relationen $(1891)^{16}$. Il est fort difficile de penser ce genre d'objet ou d'entités comme ne manifestant pas une certaine connexion où est présumée subsister en acte une complexion qui la fonde. Le fait qu'elle soit là est dépendant de ceci que ses éléments sont connectés de façon adéquate dans la "présentation" qu'on en forme. La preuve en serait que des inadéquations sont d'ailleurs "possibles" de par la structure du superius, que ce soit dans l'imagination et la mémoire. Dans l'école de Graz, Rudolf Ameseder a développé cette optique de la " production » qui fait « apparaître » la présence de la connexion sous la forme du Realkomplex. Pourtant le Relat, dit-il (la relation ajoutée) n'est pas un objet de plus. ${ }^{17}$

Les objets d'ordre supérieur supposent qu'une part de leur être soit un "être-présenté » (relevant typiquement d'une Erfassung, dit Meinong). Ils sont des objets appréhendés, bien qu'il ne s'agisse pas de l'appréhension directe au sens que lui donneront ses objecteurs pour la déconsidérer empiriquement. Ce qui vient de leur présentation intentionnelle entre dans leur constitution et ainsi s'apparente - dirait Bergmann - à un «pseudo-nexus ». Mais il ne s'agit nullement d'une constitution arbitraire ou psychologique de représentations mentales, même si la problématique est héritée des puzzles psychologiques qu'étudie Meinong dans le cours de ses analyses antérieures sur le nominalisme et les relations depuis les HumeStudien. ${ }^{18}$ Ce qui donne leur relief et leur couleur propre à ses positions l'est en vertu de son intérêt persistant pour l'abstraction et l'analyse : elle lui permet d'interroger sous plusieurs aspects différents la manière dont doivent être obtenus des « contenus » - de par leur nature et leur structure - et ce afin d'expliquer la possibilité des opérations qu'ils permettent. Des questions multiples suggérées par la notion débattue de Gestaltqualität est née une ontologie des objets d'ordre supérieur : elle suppose des fundamenta ou inferiora "donnant lieu » à la production nécessaire d'un superius. Cette opération sans doute ne se fait pas sans l'esprit, mais elle ne se fait pas non plus réductivement dans l'esprit: elle n'est jamais une représentation mentale « associée ». Il s'agit d'une sorte d'objet pour l'esprit, bien qu'il ait

\footnotetext{
16 : Nous avons montré cette différence entre la complexion gestaltique et le complexe ressemblant dans JeanMaurice Monnoyer, « Formes de complexion, types de connexion », Philosophiques, Vol 26, n², août 1999, pp. 245-261.

${ }^{17}$ : « Über Vorstellungsproduktion », in A. Meinong (ed.), Untersuchungen zur Gegenstandstheorie und Psychologie, Leipzig: Barth, 1904, P. 483.

18 : Hume Studien I. Zur Geschichte und Kritik des modernen Nominalismus - Sitzungsberichte der Kaiserlichen Akademie der Wissenschften. Philosophisch-historische Klasse, vol. 78, Wien 1877 pp. 185-260. (Gesamtausgabe vol. I, pp. 1-72) ; Hume Studien II. Zur Relationstheorie - Sitzungsberichte der Kaiserlichen Akademie der Wissenschften. Philosophisch-historische Klasse, vol. 101, Wien 1882 pp. 573-752. (Gesamtausgabe vol. II, pp. 1-172).
} 
aussi des propriétés contraintes par l'imposition de qualités du réel, et qu'il acquière comme tel une existence objective. L'être de tels objets est dépendant de la possibilité de leur saisie, qui fait qu'à proprement parler ils sont des « être-objets ». C'est bien là ce que Findlay ${ }^{19}$ a pu appeler un fort tropisme phénoménologique, tout en ne voulant pas dire que Meinong fasse une phénoménologie descriptive : il est plutôt le premier à reconnaître que la dimension qu'on prétend exclusivement descriptive risque de devenir futile (comme il le constate chez Husserl lui-même) et qu'il faut y associer une dimension « dialectique ». Ces entités sont à l'évidence moins caractérisées par une « existence » superflue, au sens que Bergmann peut conférer à ce terme, que par ce que Meinong appelle primitivement « l'être-donné » (Gegebenheit) ${ }^{20}$, soit la propriété universelle des objets d'être des objets. ${ }^{21}$ La différence d'avec le jargon de la « donation » est que ce soit des objets et d'eux seuls dont nous parlons.

Bergmann, qui reconnaît le tempérament phénoménologique de Meinong, le traduit assez fidèlement en examinant ses propositions dans leur visée ontologique, tout en s'inquiétant de savoir comment l'on peut ainsi fonder l'être des objets qui peuplent son monde. Or les entités dont traite Meinong ne peuvent aucunement jouer le rôle de «constituant» ontologique que leur confère Bergmann.

La relation chez Meinong est certes bien là : elle est ce qui se trouve être co-présent lorsqu'il y a un complexe intégré, mais elle est aussi ce qu'elle est par surcroît, comparée à une collection objective, précisément parce que cette relation fait défaut à la seconde. Cet ajout n'est rien que la conséquence analytique qui apparaît dans l'ordre d'une complexion, ou qui « doit » être là pour que l'objet soit « donné » en tant qu'objet unitaire ou comme l'un de ceux qui ne subsistent pas. L'objet révèle la complexion ou bien, si ce n'est pas le cas qu'une complexion soit obtenue, elle en fait son objet « inexistant». La relation n'est donc pas, en résumé, une entité posée dialectiquement en vue de déployer l'ontologie d'un complexe. Cela reviendrait à pouvoir rendre raison par construction du caractère intégré ou dissociable d'un complexe, comme le fait Bergmann lorsqu'il souligne dans Realism qu'un complexe existe s'il se compose d'un universel, d'un particulier brut, et éventuellement de relations entre entités qui seraient des particuliers parfaits, plus un nexus d'exemplification qui les « lie ». Quand l'ensemble est ainsi inventorié en une série d'entités dont on décrit les rôles, l'ontologie se fait sous forme dialectique.

Mais la manière dont Meinong déduit la présence de la relation en question ne repose pas sur ce genre d'approche : la relation «de plus » est présentée via le complexe donné dans une appréhension phénoménologique. Elle n'est en rien la condition ontologique qui fait qu'il y a une connexion, au sens de ce qui la produit. Meinong ne se place pas dans une optique où la position d'une entité est là pour expliquer dialectiquement ce qui doit être pour contribuer à faire exister une entité. La manière dont les objets sont appréhendés par l'esprit ne résulte pas $\mathrm{du}$ processus faisant que le superius surgisse - dans certaines conditions pré-existantes « sur » des inferiora. La complexion n'est pas produite ipso facto, a-t-on envie de dire. On comprend à cet égard que Bergmann puisse se référer plus tard à Meinong à propos des circumstances. Comme la connexion est toujours supposée pour que l'objet supérieur soit tel qu'il est en tant qu'il est, et parce qu'il est intended to, la seule disponibilité dialectique est de parler d'une certaine relation entre les constituants. Mais elle ne coïncide que

\footnotetext{
${ }^{19}$ J. N. Findlay, « Meinong the phenomenologist », Revue Internationale de Philosophie 27, pp. 161-177, 1973.

${ }^{20}$ : Meinong, Über Gegenstandtheorie, in Untersuchungen zur Gegenstandstheorie und Psychologie, op.cit., p.

20. Gesamtausgabe II, p. 500.Trad. Fr. par J.-F. Courtine et M. de Launay, in A. Meinong, Théorie de l'objet et présentation personnelle, Vrin, 1999, p. 83.

${ }^{21}$ : Ce point a été étudié soigneusement par F. Nef dans l'Objet quelconque, Paris, Vrin, 1999.
} 
« partiellement » avec la connexion, nous rappelle Meinong. Le geste dialectique qui marque la présence de la relation est là pour traduire le fait que l'entité avec laquelle elle se donne (c'est-à-dire par laquelle elle est pensée) existe dès lors que les constituants sont connectés. La Komplexion n'est pas là pour fonder ontologiquement la connexion elle-même. C'est bien ce qui explique que Meinong se dispense de l'introduction d'universaux.

Si l'analyse nous rend présente une « relation » entre constituants, celle-ci ne se confond pas avec une disposition référentielle : en revanche, si le complexe n'était pas intraconnecté ou pseudo-connecté par la pensée et s'il n'était qu'une collection objective, la relation en question ne serait pas disponible pour l'analyse et elle ne serait pas présentée comme l'ajout caractéristique des complexes intégrés.

\section{3/ Le problème de Bradley et la confusion des contextes}

Pour confirmer l'hypothèse précédente, considérons l'attitude de Meinong face au problème de Bradley. S'il pensait que la relation « ajoutée » était le facteur ontologiquement pertinent pour fonder la connexion, il aurait dû y renoncer, car loin de permettre de résoudre - dans l'ontologie - le problème de Bradley, elle le conditionne. La solution de Meinong n'est pas de le résoudre au moyen de la détermination de certaines entités (comme un nonrelational tie, ou un nexus). Dans le cas de Meinong, le problème est évité parce que le statut de la relation ne requiert pas qu'il soit résolu.

On sait que dans les textes visés par Bergmann, ${ }^{22}$ Meinong introduit le problème en question à partir de la « coïncidence » entre relation et complexion (dont le principe est écarté par Bergmann d'un revers de main $^{23}$ ). Elle signale une double dépendance : les relata sont dépendants envers la relation comme la complexion est dépendante envers la relation. Meinong refuse le caractère problématique de la régression à l'infini, ce n'est pas qu'il pense qu'elle n'est jamais vicieuse : il l'utilise lui-même comme argument dans d'autres textes ${ }^{24}$.

22 : Dans ce cas surtout, Über Gegenstände höherer Ordnung und deren Verhältnis zur innehren Wahrnehmung, op.cit.

23 : Précisément parce qu'il lui paraît faire fi du problème qu'il se pose en termes dialectiques : comment la relation peut-elle connecter? On a vu que le principe de coïncidence signale que s'il y a relation, alors il y a connexion, mais c'est dans un tout autre sens que celui que cette proposition recevrait à l'intérieur de la dialectique bergmannienne. Voir par exemple Realism, p. 370.

24 : Comme par exemple dans «Abstrahieren und Vergleichen » (1900, Gesamtausgabe I, pp. 477-8) où il réfute un argument de H. Cornelius qui consistait à montrer que l'appréhension de propriétés — d'une manière voisine de la thèse basique du nominalisme de la ressemblance - suppose de détecter des ressemblances en vertu desquelles la propriété peut être obtenue. Meinong y soutient en effet que si l'appréhension suppose la ressemblance, appréhender le résultat de la ressemblance suppose que ce résultat lui-même soit donné en vertu d'une autre ressemblance, laquelle a lieu entre ce résultat et une autre, ce qui, pour donner lieu à l'appréhension ce nouveau résultat, suppose encore qu'une ressemblance de plus soit à nouveau l'opérateur de donation, et ainsi ad infinitum. Dans ce cas, la régression est considérée comme vicieuse. Dans Über Gegenstände höherer Ordnung, le fait que la régression soit réputée non-vicieuse implique quelque chose de très différent. G. Bonino, "Why There Are No Facts in Meinong's World (according to Gustav Bergmann », Meinong Studien, Vol. II, Ontos Verlag, pp. 239-266, a tenté une reconstruction hardie dans la seule ontologie de Realism, pour justifier herméneutiquement de la position de Bergmann. 
Pour garantir que la liaison en question est organisée et intégrative, Meinong affirme que les relata se tiennent dans ce que Armstrong appellerait une certaine " direction » de la relation. $C^{\prime}$ est ce qu'il exprime en disant que $\mathrm{A}$ et $\mathrm{B}$ sont en rapport avec $\mathrm{R}$ d'une manière précise, impliquant qu'il y ait une relation $\mathrm{r}^{\prime}$ entre $\mathrm{A}$ et $\mathrm{R}$, et une relation $\mathrm{r}^{\prime \prime}$ entre $\mathrm{B}$ et $\mathrm{R}$ : ces relations $r^{\prime}$ et $r^{\prime \prime}$ pouvant éventuellement être identiques. Leur fonction est de garantir que A et $B$ sont liés correctement à $\mathrm{R}$, autrement dit, que la relation $\mathrm{R}$ « accroche » ses termes d'une manière propre à organiser le complexe comme il doit l'être. Meinong remarque que la même question se reposerait pour les nouveaux complexes relatifs qui sont formés par Ar'R et Br"R : euxmêmes pourraient être supposés impliquer des relations supplémentaires. Le problème de la régression dès que posé, se trouve donc écarté. La relation en question possède des relata dont la nature est moins de jouer un rôle "constitutif » dans la connexion que de signifier indirectement que la complexion existe, ou qu'il y a un ordre dans lequel elle consiste. Cet ordre structural est le seul corrélat pensable de la connexion. Bergmann reprend justement cette idée dans New Foundations, à propos des questions centrales de la diversité et de l'ordre internes aux « canons », dont la structure est toujours celle des circumstances.

Meinong soutient que ces relations (ordre et diversité) peuvent bien être vues comme essentielles sans que cela ne fasse d'elles des parties constituantes de la complexion. Et il est clair que l'ordre des constituants peut être compris comme supposant ontologiquement une infinité de relations essentielles en ce qu'elles construisent la nature des constituants. Mais alors le tout de ces relations ne peut fonder ontologiquement la complexion dans sa diversité. Une autre possibilité est d'envisager que la démultiplication des relations trahisse une difficulté de la fondation ontologique, auquel cas nous n'aurons plus besoin de trancher l'alternative précédente.

L'argument de Meinong consiste à dire que le caractère inadéquat de la présomption des relations supplémentaires ou constituantes se manifeste dans la «nature » de ce qu'elles tentent de relier: elles ne peuvent être conçues dans ce cas autrement que comme reliant d'autres relations. Elles ne peuvent plus jouer le rôle « constituant » que l'on est tenté de leur conférer lorsqu'on les multiplie. Meinong perçoit que ces relations supplémentaires ayant toutes pour termes une autre relation font disparaître l'objet. Ici se signale le point de retournement décisif : soit on comprend que la relation, pensée à partir de l'entité qu'est l'objet d'ordre supérieur, manifeste la connexion par le biais d'un ordre, soit on cherche à fonder ontologiquement cet ordre (et la connexion qui en est solidaire) en multipliant les relations. Le deuxième cas suppose un geste dialectique. Le premier traduit bien que Meinong ne pense pas la relation en question en termes de fondement ontologique. La relation $\mathrm{R}$ est comprise comme dépendant directement de l'ordre des parties constituantes. Cet ordre fait que le superius est là. Que certaines relations "supplémentaires » puissent être essentielles sans être constituantes traduit le sens meinongien (ou la métonymie inspirée du Daseinsfrei) affecté à la notion du « constituant».

Multiplier les relations signifie vouloir déployer à partir du donné l'ensemble des relations pensables: ce serait en faire des relations «réellement» constituantes dont l'exhaustion permettrait de fonder l'être du complexe, autrement fonder ontologiquement la connexion. Meinong choisit une autre alternative, dans laquelle on peut poser l'existence d'une relation, sans penser pour autant qu'elle « fonde » ontologiquement l'unité de ce qu'elle relie. L'erreur de la démultiplication vicieuse procède bien du souci de dialectiser « ontologiquement» ce qui ne peut pas l'être au sein d'un contexte phénoménologique, comme le reconnaît finalement Bergmann avec ces M-functions. 


\section{4/ Dualité des complexes}

Bergmann critique Meinong en affirmant de son «monde » qu'il est vide d'objets, que les choses n'y sont pas des complexes, et que s'il y a des complexes, ils ne sont pas connectés (ce ne seraient que des crypto-clusters). Mais la ligne d'interprétation précédente vaut aussi pour les « objets » ordinaires qui apparaissent comme des complexes auto-connectés, tels ceux que Bergmann assimile à des faits. Meinong prend en effet lui aussi pour exemple des objets " ordinaires », lesquels sont bien à certains égards semblables à des objets d'ordre supérieur (d'après la saisie que nous en avons) et qui sont pour cela stigmatisés par Bergmann.

Cette relation qui «s'ajoute ", même si elle est conçue par l'esprit, l'est à partir d'une certaine présentation et elle ne serait pas comme elle est si ce qui est présenté n'était pas déjà connecté. Tel est par exemple le sens de la proposition énoncée dans Abstrahieren und Vergleichen, qui souligne que le rapport d'inhérence entre A et B est toujours représentable comme une relation entre $A$ et $B^{25}$. L'inhérence traduit l'existence d'une connexion ou d'une implexion selon les cas (dans la philosophie postérieure de Meinong). Bergmann dirait alors qu'il ne s'agit que d'une exemplification du dispositif de la prédication. Mais en partant de l'appréhension d'un objet ordinaire avec ses propriétés, ce qui est dit appartenir à un objet peut être traduit par la pensée comme une relation entre l'inhérent et ce dans quoi ces propriétés « inhérent », à l'image du principe dit de coïncidence partielle présenté supra. Ce qui traduirait la forme de l'inhérence est, comme dans le cas que nous avons vu, la marque de l'intégration d'un complexe. Dans ce cas-ci non plus, elle ne le constitue pas. Dans un collectif objectif, il ne s'y trouve pas d'inferiora qui fondent un superius : telle «la croix rouge ». Si le superius n'est pas là, les inferiora sont aussi "inexistants » que lui. Pour Meinong, il y a toujours une différence entre appréhender une croix rouge, et appréhender les propriétés comme «être rouge» et "être en forme de croix». Bergmann dans les New foundations affirme de façon très voisine, abandonnant son credo de l'annexion spontanée des qualités universelles par un particulier nu, que l'objet de la présentation est la « valeur » de la M-function. Elle serait inséparable des arguments qu'elle supporte.

Comme cela ne signifie pas le besoin d'un engagement envers l'existence d'une substance ou d'un substrat, le complexe meinongien réellement «intraconnecté » reprend toute sa vigueur : nous voyons ses propriétés sous l'aspect de traits inhérents; ils se laissent analyser comme une relation entre la propriété et l'objet, et le fait qu'une qualité inhérente puisse être interprétée comme une relation hors de toute structure prédicative est de nouveau pleinement dégagé.

Sous cet aspect, l'être-ensemble (togetherness) reste une propriété phénoménologique sans avoir à fonder ontologiquement la connexion. La fonction, qui reprend du service, n'y est absolument pas vue comme une «règle de coordination» anthropomorphique. Meinong admet du reste une autre forme de complexe, appréhendé cette fois par la relation qui se tient entre ses constituants, tout en excluant qu'il soit possible de lui conférer le statut d'une fonction séparée. Il s'intéresse à des complexes traditionnels comme «x ressemblant à y » ou encore «a différent de $\mathrm{b}$ », qui ont bien un contenu phénoménologique : comment leur

\footnotetext{
25 : « Abstrahieren und Vergleichen », in op.cit., p. 469.
} 
contenu principal (ici la relation) est-il lié à ses constituants (les relata) ? On ne peut nier que cette question, là encore, ait fait l'objet de toute l'attention de Bergmann, après le silence qui suit Realism.

Faudrait-il penser en somme que la relation de ressemblance doive être reconstruite comme une entité constitutive du complexe ? Meinong parle en effet de l'objet qu'est la relation, et surtout de l'objet d'ordre supérieur qu'elle représente. Il est vrai que l'on ne peut la séparer de ses constituants, en faire une fonction détachable qui prendrait ou ne prendrait pas des arguments. Meinong lui prête en effet une sorte d'insaturation ou d'incomplétude interne (innerlichen Unfertigkeit) $^{26}$. Il y a bien ici une dépendance de ce qui est fondé en regard de ce qui fonde. Mais Bergmann la conçoit, tout au contraire, comme la marque d'une absence de fondement ontologique. Puisque la fonction implicite - qui tient lieu de la relation - est elle-même dépourvue de fondement, elle ne connecte rien. Il faut faire remarquer néanmoins que la dépendance « interne » que met en avant Meinong n'épuise pas l'être de l'objet d'ordre supérieur : il est très possible de concevoir que «l'être différent» soit inséparable de $a$ et de $b$. En tant que complexe intégré, c'est encore à travers la présentation qu'on en a que Meinong valide l'identité de ces complexes fondés sur les " natures » de $a$ et $b$, lesquelles permettent qu'un objet d'ordre supérieur soit appréhendé eo ipso (la formule est, on le sait, directement inspirée d'Ehrenfels). Mais si l'absence de fondement ontologique que mentionne Bergmann était pertinente, il n'y aurait nulle différence entre le complexe « a est différent de $b »$ et « $a$ et $b$ ». Cette exigence meinongienne de différenciation a d'ailleurs été très largement revalorisée par $\mathrm{H}$. Hochberg à la suite de Bergmann.

Bergmann affirme dans Realism que les fonctions ou bien sont à elles-mêmes leur propre fondement ontologique, ou bien «représentent» ce qui par ailleurs serait leur fondement ontologique. Frege rentre dans le second cas, Meinong dans le premier. Mais pour paraphraser le Bergmann de New foundations, et si l'on tient à la désigner ainsi, la fonction meinongienne a ceci de propre qu'elle n'est jamais dissociable et de sa valeur et de ses arguments. D'où son renouveau d'intérêt dès que la puissance connectrice du tie est battue en brèche ${ }^{27}$.

L'objet d'ordre supérieur est dépendant des termes qui le fondent, mais il ne se réduit pas, avons-nous dit, à une relation interne, ni aux représentation mentales que l'on associe à une relation. Bergmann, en vertu de l'absence de fondement ontologique propre aux relations internes, doit alors inscrire une nouvelle entité à son inventaire lorsqu'il s'intéresse à cette

\footnotetext{
${ }^{26}$ : « Beiträge zur Theorie der psychischen Analyse », Zeitschrift für Psychologie und Physiologie der Sinnesorgane. Bd.VI. 1894. Reproduit dans Gesamtausgabe I, p. 323.

27 : Ce qui importe ici est moins la dialectique de la relationnalité que l'obtention d'un fondement qui postule une connexion sans renvoyer à une entité cachée. Ce trait analytique est meinongien et relève de la mise entre parenthèses du rôle dialectique du «tying». Dans NFO, l'échange entre Bergmann et son interlocuteur « relationnaliste» le montre bien : plutôt que de chercher ce qui fonde (grounds) «l'accrochage » (clinging) (p. 128) de la fonction et de l'argument, le relationniste est invité à considérer qu'il n'y a pas de connexion supplémentaire, et donc pas de fondement de plus (specific ground) pour obtenir ontologiquement le complexe. Accepter la circonstance eo ipso de ce même «clinging » revient à le fonder. Pour Bergmann alors, et assez brutalement : «La 'connexion' devient ce que le canon dit qu'elle est » (p.132). Bergmann associe l'absence de représentativité et la nécessité du fondement de façon paradoxale, à moins de ne lui accorder que l'inscription des circonstances dans le langage idéal ne se fasse sur une base "phénoménologique ». Il accepte la thèse wittgensteinienne selon laquelle « $\mathrm{a}=\mathrm{b}$ », par exemple, ne représente rien du monde (elle réfléchit une « règle linguistique »). Mais ce qu'il nie, est qu'elle n'ait pas de statut ontologique : transcrire des intentions (des objets connus) dans le langage idéal ou canonique ne peut pas se faire uniquement en vertu de « règles linguistiques », il faut regarder le monde (Notes on Ontology, p. 145) pour obtenir et retranscrire les différences entre complexes. Inscrire les signes diacritiques dans le langage idéal reviendrait à cerner le concept de « circonstance » : certaines formes de connexité sont données en « personne propre » avec les constituants connectés.
} 
sorte de complexes «meinongiens », qui ne sont pas des complexions au sens strict. C'est le constat qu'il prononce dans la conférence de 1968, Diversity. Pour rendre raison de l'aspect phénoménologique de la différence existant entre le vert et le rouge, Bergmann admet des complexes primaires comme «l'être-différent de $a$ et $b$ » : il ne nie pas que «l'être-différent» fasse l'objet d'une présentation. En étant présenté avec le rouge et le vert, mais aussi avec leur « être-divers », il y a maintenant quatre entités : le rouge, le vert, "l'être-divers », et le complexe dont ces trois entités sont les constituants. Nous avons «l'être-différent», en plus des deux entités qui sont différentes et du fait qu'elles soient différentes. Ce qui fait quatre entités. Pour Meinong le fait que ceci et cela soient différents est identique à l'appréhension de leur différence. Cette position radicale de sa part semble n'avoir pas été maintenue par Bergmann : dans New Foundations, il affirmera de nouveau - comme Meinong - que si nous sommes en rapport phénoménologique avec "l'être-ensemble » (togetherness) de $a$ et $b$, nous désignons par là même le rapport que nous entretenons avec un fait. Le cas de ces complexes primaires révèle que la lecture des relations internes jadis opérée par Bergmann entre en conflit avec la phénoménologie du «tournant» qu'il souhaitait prendre. La reconsidération des fonctions meinongiennes et la manière dont Bergmann les distingue des relations internes dénote chez lui un mouvement conceptuel important. Il ne s'agit plus de dire qu'une fonction représente une relation.

\section{6/ Universaux : Bergmann, Russell et Meinong.}

Une critique constante de l'ontologie de Meinong est le caractère nominaliste de ses positions. Or, pour Meinong, la façon dont un complexe est donné ne requiert en rien de théoriser la nature universelle ou non de ses propriétés. Ce qui ne signifie pas que l'on doive oblitérer ce rapport: les problématiques sur les objets incomplets apparaissent déjà dans Abstrahieren und Vergleichen. Mais même leur développement tardif, comme par exemple dans Uber Möglichkeit und Wahrscheinlichkeit se produit sur un mode tel qu'il est difficile de les assimiler aux universaux de la tradition ou à ceux de Bergmann. Hormis ces approches par le biais des objets incomplets, la perspective de Meinong est qu'il convient plutôt de déterminer comment sont séparables et analysables des qualités diverses, et comment elles se partagent en inferiora et en relations.

Pour Meinong, les propriétés sont d'emblée des propriétés particulières, parce que c'est ainsi qu'elles nous apparaissent : elles sont localisées dans un complexe. Il ne fait pas de différence pour savoir si elles sont universelles ou simplement ressemblantes. Elles sont aussi bien connectées dans un fait que ne le sont les entités de Bergmann, mais Meinong adopte une ontologie propre dont l'aspect dialectique est très fortement freiné par le régime de l'appréhension qui gouverne ses recherches. Sa phénoménologie réaliste est aussi la raison (curieuse) qui veut que Meinong soit jugé d'abord par Bergmann comme "nominaliste ». Meinong rappelle certes dans Abstrahieren und Vergleichen que le chercheur qui découvre que le diamant a la propriété d'être consumé en raison de sa complexion carbonique, opère sur tel «morceau particulier» de diamant. Cela n'empêcherait pas de penser que toute entité qui a les mêmes propriétés que ce morceau de diamant présenterait la même disposition à se consumer. Mais cela ne requiert pas non plus de poser un universel instancié dans un particulier brut. On n'a pas ici besoin d'un universel et du geste dialectique qui lui est associé pour construire l'objet complexe structuré par le carbone. Si cette différence est préservée entre Bergmann et Meinong, malgré l'évolution du premier, elle souligne plutôt un décalage 
du rapport entre contexte phénoménologique et contexte dialectique dans leurs perspectives réciproques. Les rapports du mode phénoménologique et du mode dialectique seraient bien décisifs en ontologie, commandant une partie des raisons qui motivent l'introduction d'entités ou qui la limitent.

Est-ce à dire que le motif phénoménologique interdise l'appréhension d'universaux ? Cela reviendrait à dire que la postulation d'universaux est essentiellement dialectique. Mais ce n'est pas le cas. Ce problème, comme celui de l'individuation des propriétés qui en est le corollaire, relève d'une indécidabilité foncière. Sur les problèmes précédemment évoqués, où les critiques de Bergmann peuvent être relativisées en regard de l'approche meinongienne et qui semble avoir eu, si nous lisons bien, une deuxième influence sur l'ontologie finale de Bergmann -, la question des universaux demeure quasiment inchangée pour sa part. Bergmann, à l'occasion de la publication de An Inquiry into Meaning and Truth de Russell, pouvait formuler une exigence, qui se maintiendra jusque dans NFO sous la forme d'un principe. En effet, déjà dans Russell on Particulars, il s'oppose à la théorie du faisceau d'universaux de Russell qui aurait le défaut de libérer les universaux des particuliers qui les « localisent » lorsqu'ils sont instanciés. Bergmann remarque :

«There is, first, a difficulty concerning the psychological, or as I would rather say, the phenomenological status of Particulars. As it is usually put, the point is that the content of even the simplest awareness is never one or, in the relational case, several quid, but always, wether property or relation, a quale, that is, a Universal. Against such views one could defend the unqualitied particular by pointing out its indispensability in fixing the location of qualities $\gg{ }^{28}$

Ce point marque un retour de Bergmann à l'acquaintance avec les universaux revendiquée par Russell, mais celle-ci est assortie de la nécessité de ce qui apparaît comme la première formulation du particulier brut, ici un « unqualitied particular ", plus tard 1' "individuator » de New Foundations. Dans ce dernier ouvrage, nous retrouvons, plusieurs décennies après la formulation de ces quelques lignes, l'énoncé du principe dit « Principe d'Acquaintance » :

« No act intending a universal, say, $f_{1}$, occurs in a life history unless there is a particular, say, $a_{1}$, such that $f_{1}\left(a_{1}\right)$ is also intended, either by this act or by a second act, either simultaneous with or preceding the first, that occurs in the same life history $\gg .{ }^{29}$

Bergmann précise bien qu'il n'est pas identique avec le Principe de Présentation, selon lequel tout ce qui fait l'objet d'une intention existe, i.e : tout ce qui est présenté existe. Ce dernier principe est on ne peut plus meinongien. Le premier est russellien, modulé par l'exigence de la présence corrélative d'un particulier. Dans Ineffability, Ontology and Method, Bergmann présente cependant l'acquaintance et la présentation comme synonymes :

«Each of us is acquainted with some things and facts (states of affairs). Synonymously, these things and facts are presented to us $\gg .^{30}$

Or la présentation et le principe d'Acquaintance sont dissociés dans New Foundations d'une manière telle que, paradoxalement, le principe meinongien de présentation «croisant» le principe russellien d'Acquaintance joue contre Meinong, en affirmant que nous sommes en

\footnotetext{
28 : Russell on particulars, 1947, in G. Bergmann, The metaphysics of logical positivism, Longmans, Green and co., 1954, p. 200.

${ }^{29}$ : New foundations of ontology, p. 65.

30 : The Philosophical Review, Vol. 69, No. 1, Jan., 1960, p. 18.
} 
rapport non pas avec des propriétés particularisées, mais avec des universaux qui sont individués par des particuliers qui n'ont pas d'autres qualités.

Cette acquaintance avec un quale, c'est-à-dire avec un universel, est entrée très tôt dans la pensée de Bergman pour n'en plus sortir, malgré quelques modifications dont nous avons montré qu'elles s'effectuaient en profondeur entre Realism et New Foundations. Le « tournant phénoménologique » mentionné au chapitre II des New Foundations ne conditionne pas la mise à l'écart des universaux. Cette position de Bergmann remonte à ces rapports critiques avec Carnap sur la nature des « intensions » que nous ne pouvons développer ici.

Nous avons parlé ci-dessus d'une forme d'indécidabilité de la question de l'acquaintance avec des universaux (doivent-ils être appréhendés sur une base phénoménologique ou sur une base dialectique ? et n'est-ce pas un faux débat). Ce qui est certain dans les New foundations of ontology, c'est que la confirmation de l'engagement envers l'existence des universaux et la modification de la problématique de la constitution feront encore bon ménage. Une dyade unit eo ipso un individuateur et une "sorte» ultime, en lieu et place d'un nexus assurant l'exemplification d'un universel par un particulier brut. L'ontologie du lien a changé, non pas chez lui l'ontologie des constituants. La résurgence de thèmes meinongiens n'amène donc pas à un renoncement aux universaux. Russell reste beaucoup plus influent que Meinong sous ce rapport. Sur le plan interprétatif, il resterait à prouver dans un autre travail que la position de Meinong supporte le caractère « indécidable » de la postulation d'universaux in rebus.

\section{5/ Fondement ontologique et "phenomenological turn" "}

Le critère de la réapparition dans la late ontology d'aspects meinongiens qui étaient parfois complètement absents de la critique de la middle ontology, révèlent-ils certains des traits « structurels » de l'évolution de la philosophie de Bergmann?

D'une part, les traits de la pensée de Meinong qui résistaient à la critique de Bergmann en 1967 apparaissent maintenant comme des positions positives au sein du système de 1992. Dans Realism, ce qui se passe peut être décrit comme une traduction dans l'ontologie pure de ce qui chez Meinong ne lui est pas exclusivement redevable. Cela indique une priorité dialectique pour le Bergmann de l'époque : elle ne pouvait se traduire que par l'affirmation de la défaillance de l'ontologie de Meinong. Au contraire, les aspects que nous avons tentés de mettre en évidence (comme les ressorts du refus chez Meinong de la régression de Bradley, le mode d'intelligibilité que les objets d'ordre supérieur offrent pour la connexion, le sens de la relation dans les complexes et complexions) révèlent d'autres traits qui échappent à la critique de Realism et sont analogues avec certaines positions décisives de New Foundations.

Sous cette dimension nouvelle que les positions de Meinong pourraient recevoir à l'encontre de la machinerie de la middle ontology (le monde de Meinong étant incompatible avec le foil), il n'est alors plus vraiment surprenant de cerner dans New Foundations l'intérêt que reçoivent les M-Functions. Cette apparition coïncide avec une dépréciation du tie, jugé finalement absurde et phénoménologiquement problématique : 
given, there is no third. (...) $(\alpha, \beta)$ needs no other grounds than $\alpha$ and $\beta$ themselves and their each being what they are; or, as I shall also say, a diad needs no other ground thant the «identities » of its terms. Still differently, $\alpha$ and $\beta$ being there, $(\alpha, \beta)$, is eo ipso there ${ }^{31}{ }^{31}$

Bref, c'est la valeur de la fonction qui fait maintenant l'objet de la présentation phénoménologique. Rappelons-nous dans Realism, le désastre des function ontologies tenait à ce qu'elles étaient à elles-mêmes leur propre fondement ontologique. Dans New foundations, les fonctions meinongiennes sont présentées comme «la clé de la notion du fait » :

"...the fact is neither the collection, nor the class, nor the "sum » or anything else " of » the first and the second, but rather the third which is eo ipso there if and only if the first and the second are there. Or, with a twist, the nature of the fact is completely exhausted by the first satysfying the restriction which goes with the second and by the second being inseparable from the first. That is the key to the notion of fact $\gg .^{32}$

Il y a bien une relativisation du besoin de fondement ontologique pour certaines entités dont il était précédemment essentiel qu'elles en possèdent un.

Dans Realism, le tie marquait ontologiquement la différence entre le complexe formé par telles qualités de rondeur et de rougeur qui constituent un spot, et le complexe formé par ces qualités concurrentes d'ovalité et de bleu qui ne constituent rien en tant que telles, en dehors du spot. La compréhension du fondement ontologique de cette différence est présenté dés l'ouverture de Realism comme le sujet de l'ontologie ${ }^{33}$. Le nexus était l'entité-solution pour la question du fondement ontologique des faits. Bergmann soutenait déjà pourtant que l'idée d'une différence de degré dans le mode de présentation phénoménologique implique une différence catégorielle dans l'ontologie. On le voit dans la manière dont il répond à un objecteur phénoménologue imaginaire dans Realism, celui-ci pense que le lien entre ontologie et phénoménologie est trop fort : il exige des présentations phénoménologiques auxquelles il est difficile de souscrire, comme une présentation « multiple » des entités. L'objecteur affirme que ce que propose Bergmann rend la croix phénoménologique trop lourde à porter :

« it is phenomenologically absurd, as absurd as anything could be, to maintain that, the two things $e$ and $c$ being what they are, the former would not be higher than the latter unless there were also, in addition to these two, a third thing, of the kind you call relational, and still another entity, of the kind you call nexus, and unless this fourth entity tied the other three, which you hold to be « completely external » to each other, into a « fact ». ${ }^{34}$

A cette objection Bergmann rétorque qu'il n'en est rien, et que la phénoménologie en question est scandée par autant de différences et modulations « in feel ». Il affirme : All this is phenomenology without torture :

«I distinguish sharply between the phenomenological and the dialectical context. Sometimes, when simultaneously presented with two pitches, I am also, strikingly, lucidly, and without any torture, presented with the interval as well as with the fact that it connects (obtains between) the pitches. To insist on that, as I do, is not to deny, as I don't, that the several entities presented of

\footnotetext{
31 : New foundations, p. 68 et p. 105.

32 : Ibid, p. 129.

33 : Realism, p. 6

${ }^{34}$ : Ibid., p. 68.
} 
this sort differ in «feel». (...) One might wish to say, for instance, that while a pitch is more «palpable» than an interval, the latter is in turn more «palpable » than exemplification (...) Dialectically, my assay reflects the phenomenological différences between the several entities by assigning them to several categories witch differ in ontological status $\gg{ }^{35}$

L'exemple de Bergmann est la différence de hauteurs entre deux sons (plus palpable que celle de l'intervalle, elle-même plus palpable que celle de l'exemplification). Il le confronte avec le fait que Peter soit assis sur une chaise. Il y a toutefois une différence dans la manière dont ces deux faits sont l'objet d'une présentation. Elle se reflète dans la distinction dialectique qu'il opère : les entités sont associées a priori dans le premier cas, dans l'autre la chaise et Pierre sont «adventices » l'une à l'autre, la circonstance est contingente. Le phénoménologue qui ne pratique pas ces différences in feel à partir de la différence entre les catégories les plus simples ne peut pas rendre compte de la différence entre ces deux faits. Bergmann soutient ainsi que la catégorie de l'entité qui joue le rôle de fondement varie en correspondance avec la différence de présentation phénoménologique. Plus exactement, les éléments reliés, la relation ou le nexus, plus le "fait» lui-même ne font pas l'objet d'une présentation phénoménologique multiple, ni qui serait de même degré d'une étape à l'autre.

Or, le déplacement théorique qui suit Realism corrige cette proposition. Bergmann ne pense plus que le nexus nous soit donné, fut-ce de manière peu «palpable ». La présentation d'un fait n'est pas la présentation d'un fait plus celle d'un nexus. C'est un être-ensemble qui nous est présenté (ce que Bergmann dans Realism voyait comme la présentation du nexus « et » du fait). Nous sommes renvoyés à la valeur d'une fonction dont le complexe est inséparable. C'est donc cet être-ensemble qui prend le statut d'une entité au sens plein à travers la problématique des circumstances : il n'y a plus d'autre fondement ontologique que le fait que l'entité complexe soit obtenue, sans nexus, mais parce que la connexion est circonstanciée et soutenue par un acte.

C'est pourquoi dans New Foundations, la pensée de la connexion se résorbe dans celle d'une fonction, traduisant ontologiquement ce qui dans la sphère phénoménologique est présenté comme un «être-ensemble». De même encore, dans ses Notes on Ontology ${ }^{36}$, Bergmann estime qu'il est requis de procéder à certaines notations dans le Langage Idéal de manière à éviter la suggestion d'un Tie. L'exemple porte cette fois sur un donné basique "this-being-green », qui se laisse décrire comme la présentation de deux choses avec celle de leur être-ensemble ( togetherness »). Une notation possible dans le langage idéal est celleci : g1(a). Mais sa formulation linéaire suggère toujours un tie.

Bergmann explique qu'il convient en effet de partir de la dyade (g1, a), mais de conférer le statut d'une entité (to " ontologize ») à l'être-ensemble, non pas sous l'aspect d'un lien, mais comme un sous-détérminé ( $\eta$ ) qui, par sa dépendance, s'accroche (" clings ») à un déterminé unique : la dyade ( $\mathrm{g} 1, \mathrm{a})$ étant toujours dans ce cas le seul déterminé approprié. Il faut donc écrire dans le langage idéal non pas ( $\mathrm{g} 1, \mathrm{a})$, mais $\eta(\mathrm{g} 1$, a). La façon dont nous est présenté l'être-ensemble demande d'éluder une entité du type tie, ou encore maker - pour recouvrer le contexte phénoménologique de la «togetherness » dont Bergmann dit de sa présentation qu'elle est identique avec celle d'un fait.

En résumé, cet « être-ensemble » est donné, mais il l'est en même temps que la valeur d'une fonction inséparable de lui. Le caractère eo ipso de la circumstance est donc privilégié. Les

\footnotetext{
$35:$ Ibid.

${ }^{36}$ : « Notes on Ontology », Noûs, vol. 15, n² 2,1981, pp. 131-154.
} 
deux contextes se chevauchent. Ontologiquement, si les fondements sont là, le fondé l'est aussi. Mais phénoménologiquement, si une entité fait l'objet d'une présentation explicite, alors ses constituants feront l'objet d'une présentation implicite. On peut ainsi avancer que «l'être-ensemble » phénoménologique répond au caractère eo ipso du rapport existant entre ce qui fonde et ce qui est fondé : un rapport que marque la fonction, en ce qu'elle dépend des constituants sans leur être réductible. Elle demeure un sous-déterminé. Elle ne peut pas faire l'objet d'une présentation. Mais elle se substitue au nexus en tant que description de la connexion. Le tie est rejeté comme «l'absurdité d'un lien qui lie ce qu'il lie à ce qu'il lie ${ }^{37}$ ». Etre un fait devient identique à être la valeur d'une fonction ${ }^{38}$.

L'importance que reçoit désormais la notion de "circumstance » traduit bien ce décalage : telle entité est localisée lorsque telles autres sont là elles aussi. Bergmann n'a plus besoin de théoriser ce qui connecte ces entités, mais plutôt de partir du fait qu'il se trouve que certaines entités sont dans l'area de la connexion. Il privilégie finalement la thématique de la fondation sur celle de la relation, réputée donner prise au risque de l'anthropomorphisme en ontologie. Le point nodal qui permet d'écarter le phantasme d'une dialectique « phénoménologisée » est bien la présentation d'une valeur de fonction : celle-ci est tout à la fois la présentation d'un fait et la présentation de "l'être-ensemble» des constituants, sans qu'il soit plus besoin de détailler ontologiquement le fondement de cet «être-ensemble». La connexion cesse de valoir en tant que condition ontologique. Cette stance philosophique paraît elle-même fortement réminiscente du Meinong de Über Annahmen (qui étudie au $\S 40$ la contraposition stricte de Zusammensetzung et Zusammenstellung). L'évolution dernière de Bergmann semble alors moins virtuose et moins futile comprise sous cet angle. La présentation de la valeur indique que ce qui sert de fondement ontologique se réplique dans le langage idéal, tandis que la fonction " meinongienne » n'implique pas conditionnellement, ou matériellement, qu'il y ait une connexion de trop.

\footnotetext{
37 : New Foundations, p. 128.

38 : Ibid., p. 126.
} 